\title{
NLC Interaction Region Layout and Background Estimates
}

J. Gronberg, S. S. Hertzbach, L. Keller, T. Markiewicz, T. Maruyama

This article was submitted to Linear Collinder Workshop

Chicago, IL

October 24-28, 2001

U.S. Department of Energy

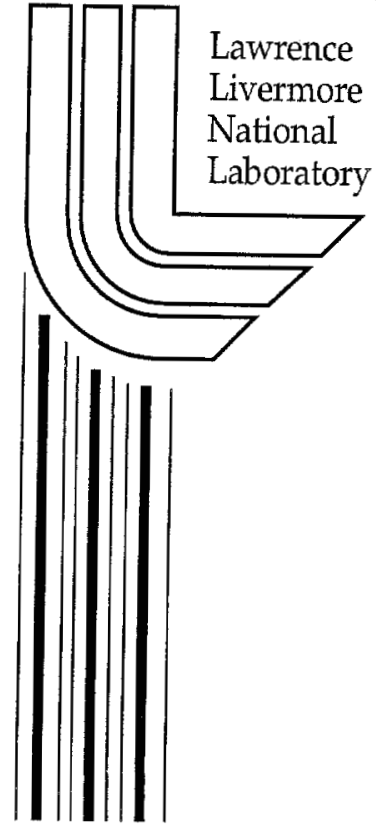

February 1, 2001 


\section{DISCLAIMER}

This document was prepared as an account of work sponsored by an agency of the United States Government. Neither the United States Government nor the University of California nor any of their employees, makes any warranty, express or implied, or assumes any legal liability or responsibility for the accuracy, completeness, or usefulness of any information, apparatus, product, or process disclosed, or represents that its use would not infringe privately owned rights. Reference herein to any specific commercial product, process, or service by trade name, trademark, manufacturer, or otherwise, does not necessarily constitute or imply its endorsement, recommendation, or favoring by the United States Government or the University of California. The views and opinions of authors expressed herein do not necessarily state or reflect those of the United States Government or the University of California, and shall not be used for advertising or product endorsement purposes.

This is a preprint of a paper intended for publication in a journal or proceedings. Since changes may be made before publication, this preprint is made available with the understanding that it will not be cited or reproduced without the permission of the author.

This report has been reproduced directly from the best available copy.

Available to DOE and DOE contractors from the

Office of Scientific and Technical Information

P.O. Box 62, Oak Ridge, TN 37831

Prices available from (423) 576-8401

http://apollo.osti.gov/bridge/

Available to the public from the National Technical Information Service

U.S. Department of Commerce 5285 Port Royal Rd., Springfield, VA 22161 http://www.ntis.gov/

OR

Lawrence Livermore National Laboratory Technical Information Department's Digital Library http://www.llnl.gov/tid/Library.html 


\title{
NLC Interaction Region Layout and Background Estimates
}

\author{
Jeff Gronberg \\ Lawrence Livermore National Laboratory ${ }^{1}$ \\ Livermore, California 94550 \\ Stanley S. Hertzbach \\ University of Massachusetts ${ }^{2}$ \\ Amherst, Massachusetts 01003-9337 \\ Lew Keller, Tom Markiewicz, Takashi Maruyama \\ Stanford Linear Accelerator Center ${ }^{3}$ \\ Menlo Park, California 94025
}

\begin{abstract}
The design of the final focus system has been modified since the previous $\mathrm{LC}$ workshop in Sitges. The system has a reduced length and the front face of the final focusing quadrupole has been moved back to 4.3 meters from the interaction point. We report the effect of these changes on detector backgrounds.
\end{abstract}

\section{INTRODUCTION}

The design of the interaction region (IR) [1,2] has changed in response to changes in the design of the final focus system [3]. Figure 1 shows the new IR layout. The front face of the final focus quadrupole has moved from 2 to 4.3 meters. It is more easily stabilized in this position since it is not as far cantilevered into the endcap of the detector. The mask, M1, has also been pulled back. It can be more easily supported in this configuration rather than the 1.5 meter cone in the previous

1) This work was performed under the auspices of the U.S. Department of Energy by the University of California, Lawrence Livermore National Laboratory under Contract No. W-7405-Eng-48. 2) Work supported by Department of Energy grant DE-FG02-92ER40715 (UMass) and contract DE-AC03-76SF00515 (SLAC).

3) Work supported by Department of Energy contract DE-AC03-76SF00515 
design. The beam pipe is still designed to respect the region where the $e^{+} e^{-}$pairs travel.

\section{BACKGROUNDS FROM $e^{+} e^{-}$PAIRS}

The changes in the final focus have not changed the distribution of $e^{+} e^{-}$pairs produced at the interaction point (IP), however the changes in $\mathrm{L}^{*}$ and masking, as seen in Figure 1, have changed the backgrounds reaching the detector. We examine the charged particles and neutrons reaching the silicon vertex detector (SVX) and the low energy photons entering the tracking volume.

In previous studies, the solenoidal field line which intersects the first layer of the SVX had an unobstructed path to a region where particles were showering. This allowed low energy particles to spiral back and create hits in the SVX. As can be seen in Figure 1, that field line now terminates on the front face of the luminosity monitor, where a layer of beryllium further reduces particle production. In this configuration back-scattered particles are negligible compared to pairs which directly strike the silicon. The hit densities are shown in Figure 2.

The pairs which strike the luminosity monitor produce photons which enter the tracking volume. By moving the aperture of the $\mathrm{M} 1$ mask from $2.5 \mathrm{~cm}$ radius at 50

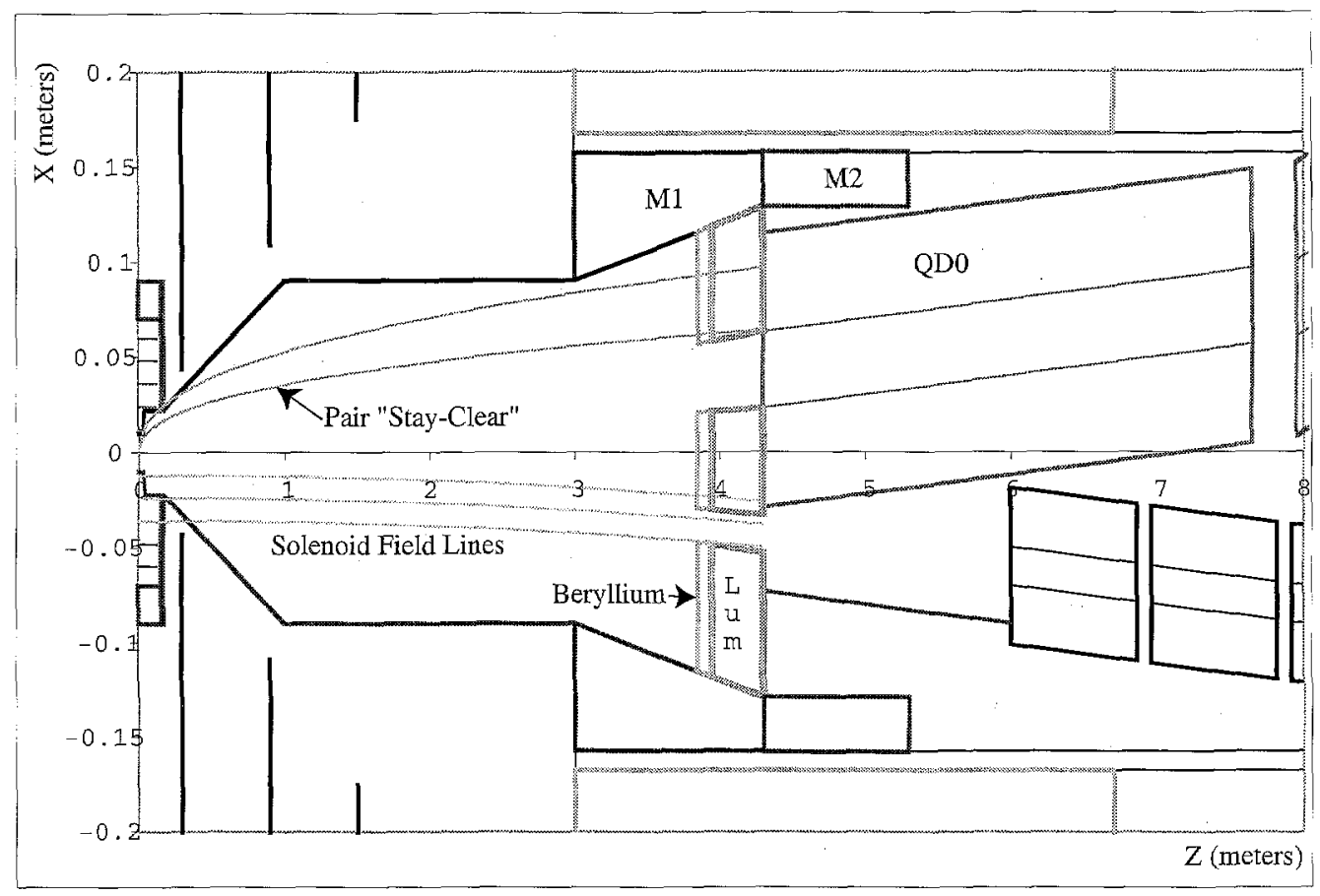

FIGURE 1. The layout of the interaction region with the large detector and a 4.3 meter $\mathrm{L}^{*}$. The orange line shows the limit of the pairs for a 4 Tesla and 6 Tesla solenoidal field. The blue lines are the solenoidal field lines which pass through the first 3 layers of the SVX. 
$\mathrm{cm}$ in $\mathrm{z}$ to $10 \mathrm{~cm}$ radius at $300 \mathrm{~cm}$ in $\mathrm{z}$ we have increased the number of photons reaching the tracking volume. However, as can be seen in Figure 2, their rate is still acceptable even for the $1 \mathrm{TeV}$ case.

In the previous IR design with a Large detector at $1 \mathrm{TeV}$ center of mass energy, the SVX received $4.1 \times 10^{9}$ neutrons $/ \mathrm{cm}^{2} /$ year from $e^{+} e^{--}$pairs showering in the luminosity monitor. The rate is reduced to $7.4 \times 10^{8}$ neutrons $/ \mathrm{cm}^{2} /$ year by moving $\mathrm{L}^{*}$ to 4.3 meters and increasing the extraction line aperture to a $2 \mathrm{~cm}$ radius to allow more particles to exit without showering. An additional reduction to $2.0 \times 10^{8}$ neutrons $/ \mathrm{cm}^{2} /$ year is achieved by adding a $50 \mathrm{~cm}$ think layer of low- $Z$ material to the front face of the luminosity monitor. This is still the dominant source of neutrons reaching the SVX. The rate for neutrons from the beam dump remains unchanged at $0.5 \times 10^{8}$ neutrons $/ \mathrm{cm}^{2} /$ year.

\section{MUONS}

At SLC startup, the Mark II detector had a serious muon backgroundapproximately 50 muons/pulse at design current. Magnetized iron spoilers were installed in the final focus tunnel, and they reduced the background by a factor of 10-100 at each source where the beam halo was scraping. For a 1 TeV CM linear collider this problem is more difficult because the muon energy spectrum is ten

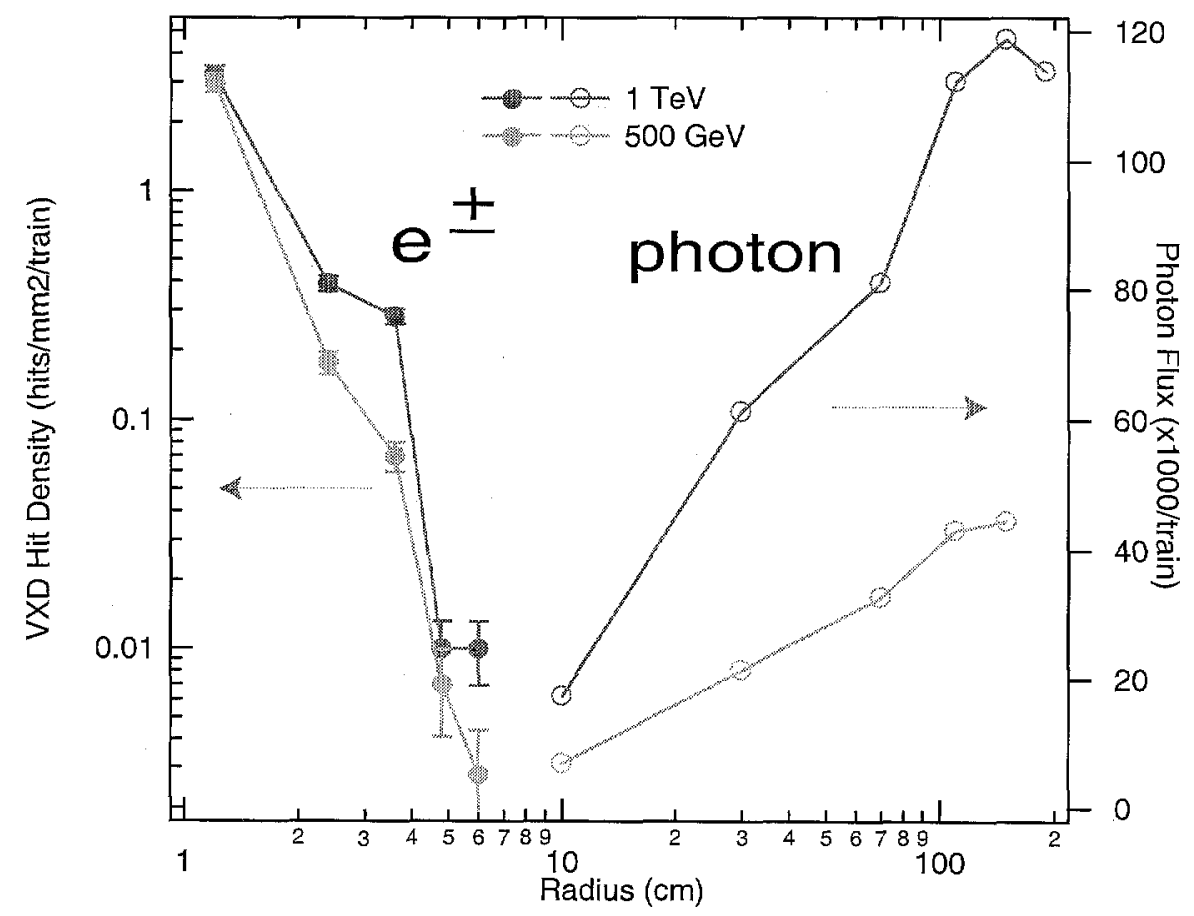

FIGURE 2. The charged hit density in the SVX and the photon density in the drift chamber for the $500 \mathrm{GeV}$ and $1 \mathrm{TeV}$ center of mass scenarios. 
times harder and the bunch train intensity about 50 times larger than in the SLC.

As shown in Figure 3 the NLC tunnel layout for this study consisted of a straight, $4.3 \times 4.3 \mathrm{~m}$ square "high energy" beam tunnel leading to a $6.75 \mathrm{~m}$ radius large detector and a branch tunnel at $26 \mathrm{mrad}$ for a "low energy" interaction region. The high energy beam line had an energy collimation section with two absorbers, followed by a betatron collimation section with eight halo absorbers, followed by the final focus to the IR. Using Program MUCARLO, muons were tracked from the energy collimation absorbers and from representative absorbers in the betatron collimation section and a high beta collimator in the final focus. For a muon to be counted as a hit in the large detector endcap or EM calorimeter, it must have penetrated one meter of iron in the end door. The muon source terms included Bethe-Heitler pair production and direct e+ annihilation in the case of the positron beam. As shown in Figure 3 two large toroidal magnetized iron spoilers were added to the tunnel to deflect muons away from the detector.

For the muon endcap and a $250 \mathrm{GeV}$ beam we examine the cases of no magnetic

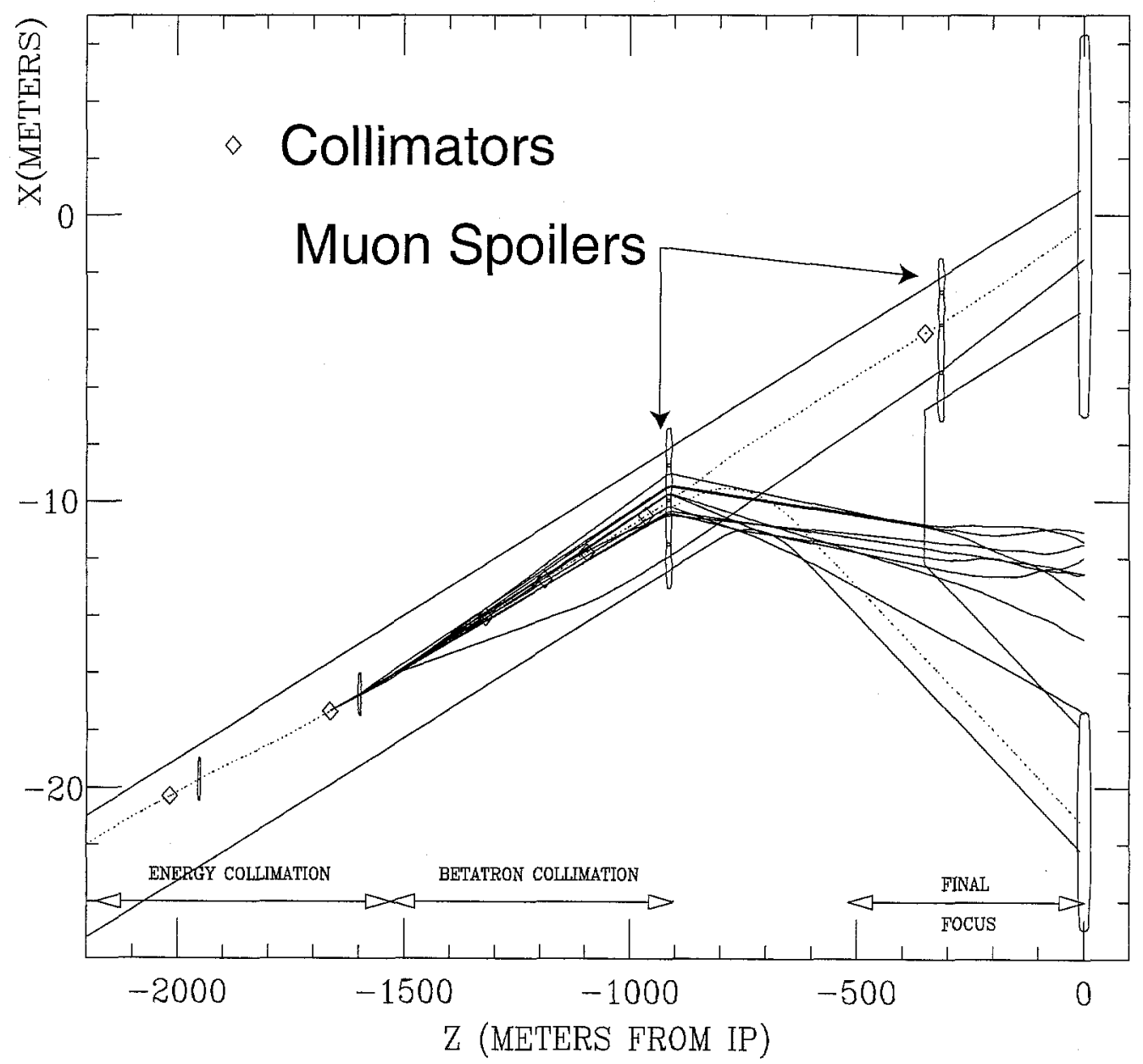

FIGURE 3. Muons produced in the collimators. 
spoilers and two full-tunnel spoilers. With no spoilers it is seen that beam losses in the range of $10^{5}$ to $10^{6} /$ bunch train in the energy and betatron collimation sections will result in one muon in the endcap. When the two full-tunnel spoilers are present, a beam loss of about $10^{10}$ in the energy collimation section and a few $10^{8}$ in the betatron collimation section will result in one muon in the detector. If the collimation system reduces the halo to $10^{4}$ on the high beta collimator in the final focus, then there will be less than one muon/bunch train in the endcap for both spoilers and no spoilers.

\section{SYNCHROTRON RADIATION}

We consider synchrotron radiation (SR) generated by the nominal Gaussian beam, and by beam halo, in the final doublet and bend magnets. SR generated in other quadrupole magnets, farther from the interaction point, is easily masked because of the intervening bends. We are primarily concerned that no SR be directly incident on the $10 \mathrm{~mm}$ radius beam pipe. Secondly, we will assure that SR scattered from masks is not a significant background source.

SR generated in the final doublet by the nominal Gaussian beam occupies a region of radius $6 \mathrm{~mm}$ near the IP. We have varied the beam energy by $1 \%$, the energy collimation range, and the beam emittance by a factor of 9 , which increases the radius of the $\mathrm{SR}$ swath at the IP to $7.5 \mathrm{~mm}$. No SR is incident on the $10 \mathrm{~mm}$ radius beam pipe, but it is unlikely that a beam pipe much smaller than this would be feasible.

If not masked, SR from the last final focus bend deposits $2.0 \times 10^{10} \mathrm{SR}$ photons $\left(k_{c}=69 \mathrm{keV}\right)$ per bunch train on the beam pipe. In the initial $4.3 \mathrm{~m} \mathrm{~L}^{*}$ final focus design, masking the bend SR is difficult because of the large beam size and the small separation $(<20 \mathrm{~m})$ between the bend and doublet. This separation has been increased to improve the masking.

Finally, SR generated by beam halo particles has been simulated for a conservative halo model which assumes $0.1 \%$ of the beam to be within the collimation aperture, in a halo characterized by a sigma 1000 times nominal. This "flat" halo model is based on experience at SLC, but contains a much larger fraction of the beam than does any calculation of halo from first principles.

The requirement that halo SR pass through the IR, and not strike the beam pipe or final focus apertures, limits the beam size in the final doublet. This requirement is achieved if the angular beam size at the IP is limited to $280 \mathrm{microrad} x 1200$ microrad. To provide some margin we have proposed collimation at 240 microrad $x$ 1000 microrad. In Figure 4 we show horizontal and vertical projections of halo SR fans for trajectories at these collimation limits. In the horizontal plane 59 microrad is required to accommodate angular dispersion and the $1 \%$ energy collimation. In each plane allowance must be made for phase migration in the $x-x^{\prime}$ or $y-y$ ' phase space. With a nominal angular beam size at the IP of 28 microrad $x 40$ microrad, the required collimation is then $4.6 \sigma_{x} \times 17.7 \sigma_{y}$. 
Y vs $\mathrm{S}$ for $\mathrm{n}_{\mathrm{X}} \sigma_{\mathrm{X}}{ }^{\prime *}=240 \mu \mathrm{rad}$

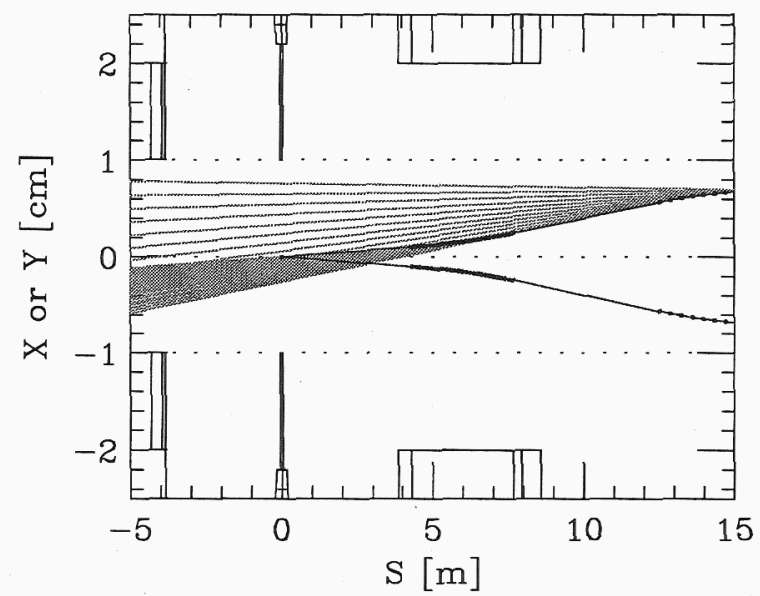

$\mathrm{Y}$ vs $\mathrm{S}$ for $\mathrm{n}_{\mathrm{Y}}{\sigma_{\mathrm{Y}}}^{{ }^{*}}=1000 \mu \mathrm{rad}$

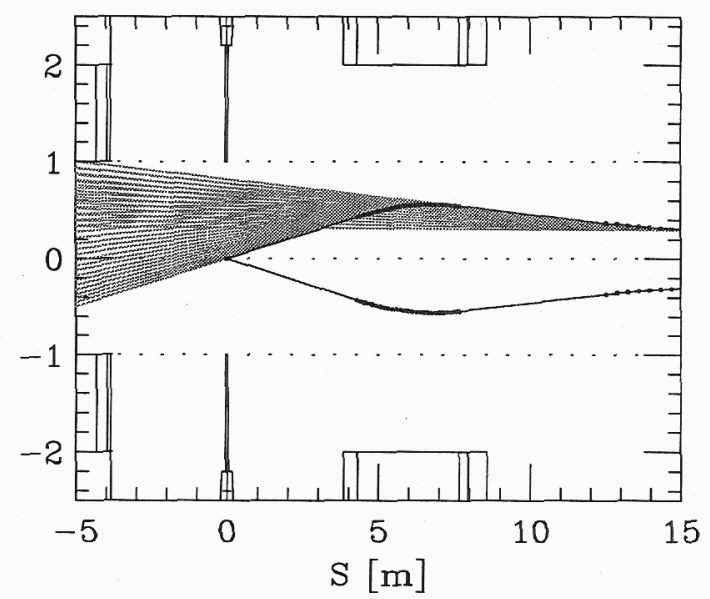

FIGURE 4. Projections of halo SR fans for trajectories making angles of 240 microrad and 1000 microrad with the beam line. For clarity the fans are drawn on only one side of the beam line.

\section{CONCLUSIONS}

The reduction in length of the new final focus degrades the effectiveness of a big bend for reducing muon backgrounds. Since the big bend is no longer important one of the interaction regions can be inline with the accelerator, leaving room for energy upgrades in the future to multi-TeV energies. The synchrotron radiation level is acceptable although the final bend must be sufficiently far away to be masked. Neutron radiation in the SVX has been reduced by moving the luminosity monitor to 4.3 meters, adding a low-Z front face, and increasing the exit aperture. The rate of charged hits in the SVX remains unchanged.

\section{REFERENCES}

1. The NLC Design Group, "Zeroth Order Design Report for the Next Linear Collider", $L B N L-5424, S L A C-474, U C R L-I D-124161, U C-414$ (1996).

2. Proceedings of the 1999 Workshop on Physics and Experiments with Linear $e^{+} e^{-}$ Colliders, to be published by World Scientific.

3. P. Raimondi and A. Seryi, "A Novel Final Focus Design for Future Linear Colliders" LCC-0039, SLAC-PUB-8460, (2000). 